\title{
Modelo de responsabilidad social universitaria: una propuesta para las instituciones colombianas
}

\section{A model of university social responsibility: a proposal for the Colombian institutions}

Martha Yaneth Forero-Jiménez'

Recibido: septiembre 10 de 2018

Aceptado: diciembre 14 de 2018

\begin{abstract}
Resumen
El artículo propone un modelo para implementar la Responsabilidad Social Universitaria, RSU, a partir de un breve análisis de su situación en Colombia. Se aplicó la investigación cualitativa, específicamente descriptiva-explicativa, utilizando el ciclo Shewhart. El modelo propuesto parte de los cuatro pilares de la educación superior: docencia, proyección social, investigación y gestión, sumando medio ambiente y posconflicto. El medio ambiente, pese a formar parte del esquema de la RS, apenas está siendo tratado con cierto rigor en la literatura, mientras que el último aspecto es de gran relevancia dada la realidad actual del país. Igualmente, se diseñó la tabla de indicadores, herramienta clave para conocer el estado de cada universidad respecto a su compromiso social y en consecuencia tomar las medidas conducentes a mejorar. Se concluye que una de las causas de la no implementación de la RSU, es que no se cuenta con una metodología o un modelo de cómo hacerlo, así como al escaso interés prestado por las universidades; esto debido a que no se ha tomado consciencia de su importancia y lo favorable que resulta para contribuir a la reconstrucción del tejido social.
\end{abstract}

Palabras clave: conflicto, indicadores, problemas sociales, responsabilidad social.

\begin{abstract}
This article is the result of an investigation whose objective was to propose a model to implement the RSU (University Social Responsibility) from a brief analysis of its situation in Colombia. Qualitative research was applied, specifically descriptive-explanatory, using the Shewhart cycle, resulting in the proposed model composed by the four pillars of higher education: teaching, social projection, research and management, plus environment and post-conflict. Environment, despite being part of the social responsibility scheme, is hardly being treated with any rigor in literature and post-conflict is of great relevance given the current reality of Colombia. In parallel, the table of indicators was designed, which became a key tool to know the status of each university with respect to its social commitment and consequently take measures to improve it. Finally, it is concluded that one of the causes of the lack of implementation is due to the lack of a methodology or a model of how to do it, as well as the lack of interest, because we have not become aware of its importance and how favorable it is to contribute to the reconstruction of the fabric of society.
\end{abstract}

Keywords: conflict, indicators, social problems, social responsibility.

1 Administradora Industrial, Estudiante de Doctorado en Economía y Empresa, Universidad Nacional de Educación a Distancia, Madrid, España.E-mail: mforero4@alumno.uned.es 


\section{Introducción}

Este trabajo contiene los elementos y la dinámica de un modelo para implementar la Responsabilidad Social Universitaria, RSU, propuesta que parte de la necesidad de las universidades de contar con una herramienta que les ayude con este propósito. Para el caso particular colombiano, es indudable que las instituciones de educación superior, dadas sus condiciones y características actuales, se ven forzadas a tener que enfrentar una serie de desafíos, no solo por los grandes cambios que se han venido causando en el plano internacional, sino también por la agudización de las crisis de los países latinoamericanos y Colombia no es la excepción.

Para analizar esta problemática es necesario mencionar alguna de sus causas, y es que uno de los inconvenientes que tienen las universidades para enfrentar tales desafíos es el poco interés prestado sobre su responsabilidad social, entre otras razones porque todavía se persiste en conservar el esquema tradicional de gestión académico administrativa y se continúan mirando las universidades en términos estadísticos; dejando además lo relacionado con programas sociales a divisiones como proyección social o extensión universitaria u otras denominaciones asignadas, lo cual no significa que esté mal sino que la RSU va más allá e involucra a toda la estructura organizativa. Consecuencia de esto, es que no se cuenta con un modelo o método que le sirva de guía para emprender la tarea, y se pueda percibir de forma tangible como se contribuye a resolver las diferentes problemáticas sociales y lo favorable que resulta directamente a la universidad.

Es necesario que las universidades reconsideren su dinámica y propendan por la congruencia institucional, que se alcanza mediante la alineación de sus funciones sustantivas, a saber: docencia, proyección social, investigación y gestión con la misión, así como la vigilancia permanente de que los efectos directos y colaterales de la actividad universitaria no entren en contradicción con los fines pregonados. En este orden de ideas, otros aspectos a considerar y que por su valor agregado se definen aparte son: el medio ambiente, que si bien no es propiamente una función sustantiva si debe formar parte de las principales actividades de la gestión institucional. Y el posconflicto que debe ser otra cuestión a tener en cuenta con igual atención, en razón a que la educación tiene el potencial de enseñar nuevos valores, actitudes, habilidades y comportamientos, al igual que puede ayudar a promover nuevas relaciones sociales que construirán resiliencia al conflicto (Infante, 2013).

Para ello, se necesita involucrar a los actores de la comunidad universitaria en un auto diagnóstico continuo del quehacer institucional, con herramientas apropiadas para garantizar la transparencia, la participación y la mejora continua de la universidad hacia su responsabilidad social, enmarcada en la búsqueda global de un desarrollo más humano y más sostenible (Yfarraguerri, 2014).

El punto de partida de la propuesta fue la mirada sobre la situación actual de las universidades colombianas a la luz de la responsabilidad social, RS, para luego a través del ciclo PHVA (planear, hacer, verificar y actuar) lograr que las actividades propias de las universidades entren en una dinámica mediante programas y proyectos interdisciplinarios. Al beneficiarse de un instrumento como el modelo propuesto, será más fácil y motivador para las universidades el emprender la tarea de implementar la RSU adecuadamente y en forma sistemática.

\section{Metodología y fundamentos del modelo propuesto}

\subsection{Metodología}

La metodología empleada en este trabajo fue la analítica-descriptiva y a la vez la explicativa; la primera en razón a que se cumplen condiciones como ... describir el estado, las características, factores y procedimientos presentes en fenómenos y hechos que ocurren en forma natural (Lerma, 2016). En 
otras palabras "describir la realidad de situaciones, eventos, personas, grupos o comunidades que se estén abordando y que se pretenda analizar ..., (y la segunda) ya no solo describe el problema o fenómeno observado sino que se acerca y busca explicar las causas que originaron la situación analizada. (Es decir), es la interpretación de una realidad o la explicación del por qué y para qué del objeto de estudio; a fin de ampliar el "¿Qué?" de la investigación exploratoria y el “¿cómo?" de la investigación descriptiva” (Universia, 2017).

Igualmente, es un estudio cualitativo pues por su naturaleza es más bien inductivo, lo cual es cierto, particularmente, si su alcance es exploratorio o descriptivo (Hernández, Fernández \& Baptista, 2003). Para el caso en particular, se da cuenta del estado de la RSU en Colombia así como de algunos lineamientos y procedimientos para su ejecución, como es el planteamiento de un modelo para este fin.

\subsection{Fundamentos del modelo}

\section{La RSU en Colombia}

En la actualidad son escasos los estudios que precisen la situación de la responsabilidad social en las universidades colombianas. En términos generales se desarrollan actividades enmarcadas en la proyección social o extensión, pero no se hace gestión de la responsabilidad social propiamente dicha. No obstante, según la Asociación Colombiana de Universidades, ASCUN (2010), el país en los últimos tiempos no ha estado tan alejado a la real dimensión de la RSU, en virtud de la generación progresiva de espacios que permiten conformar acciones de responsabilidad social, visibilizados en programas y proyectos surgidos, aplicados y sistematizados desde los contextos universitarios.

Cualquiera sea el caso, es crucial que las universidades reorienten sus esfuerzos, abandonen el asistencialismo y vayan tras proyectos más consistentes, duraderos y sistemáticos, por lo que se re- quiere de modelos o derroteros que contribuyan a hacerles más fácil su implementación.

La RSU y su relación con las actividades sustantivas de las universidades

"Resulta necesario señalar en función de lo que la RSU representa, que esta no puede ser una tarea de desempeño de la función extensión, ni vista de manera simplista como asunto de proyección social. Su enfoque es más complejo y debe abarcar de manera transversal todas las funciones propias de las Instituciones de Educación Superior, IES, a saber: docencia, investigación, extensión y gestión" (Aristimuño, 2012). Como afirma Vallaeys (2006), "la responsabilidad social no solo permea todos los procesos universitarios sino que los hace interactuar orgánicamente, incluyendo en una misma estrategia de gestión las actividades sustantivas de una universidad".

Igualmente, el medio ambiente y el posconflicto deben ser parte substancial del quehacer universitario. Está claro que "la universidad sin ser una industria de gran contaminación, tiene un impacto ambiental negativo que no se puede subestimar" (Vallaeys \& Carrizo, 2011). Además, frente al posconflicto en Colombia, la educación superior tiene el deber de preparar a los desmovilizados no solo en una disciplina, sino además hacer que se apropien valores como: justicia, democracia, tolerancia, convivencia, respeto, cooperación, autonomía, racionalidad, amor a la verdad, compasión y perdón que es de donde parte todo. "Que enseña a llevar vidas emocionalmente más saludables y que impulsa la convivencia pacífica y armónica" (Buitrón \& Navarrete, 2008, citado por Pérez, 2016).

En tal virtud, la universidad debe estar evaluando y autoregulando sus actividades en forma permanente, por lo que "La Responsabilidad Social Universitaria facilita esta autorreflexión fdinstitucional a través de las herramientas de autodiagnóstico participativo que propone. No es en sí misma un cuerpo doctrinario que se impone a las Universidades" (Vallaeys, 2008). 


\section{Importancia de contar con un modelo de RSU}

Es necesario implementar un modelo de RS en el ámbito académico en razón a que (Asociación de Universidades Confiadas a la Compañía de Jesús en América Latina, 2014):

- En la misión de las universidades está implícito el compromiso social, pero además porque se debe a la sociedad y debe procurar hacer que la gestión institucional contribuya a quitarle fuerza al desequilibrio social en que las administraciones del Estado han llevado al país en las últimas décadas.

- Para facilitar el seguimiento y autodiagnóstico de manera sistemática y periódica de la gestión de la RSU y de esta manera fomentar el compromiso de toda la institución en la gestión y aplicación del enfoque de RSU.

- Para tener insumos objetivos que faciliten la toma de decisiones.

- Para visualizar los aspectos relevantes del estado de situación y los cambios que se registran en el tiempo respecto a la institucionalización del enfoque.

- Para facilitar el análisis comparativo de los procesos, a nivel intrainstitucional (de una unidad académica o área con otras de la misma universidad) e inter-institucional (de una universidad con otras).

\section{Modelo propuesto}

“Frente a los desafíos del mundo altamente competitivo y lleno de cambios ..., las organizaciones se han visto obligadas a redoblar sus esfuerzos para ser cada día más competitivas ..., en este contexto salir al encuentro de la mejora continua se ha vuelto una necesidad" (Sarmiento, 2009). Lo cual significa que es necesaria una trasformación en la manera de pensar y actuar de las organizaciones, y el sector de la educación superior no está exento de esta afirmación. Por tal razón se plantea un método práctico y dinámico que guíe, dirija y focalice a las universidades en su compromiso con la sociedad a través del ciclo PHVA, el cual por su diseño facilita el proceso de programas o proyectos, desde su planificación, puesta en marcha, verificación de su desarrollo y correcciones necesarias. De esta manera, el modelo servirá a las universidades para replantearse en forma constante y trazarse retos a cumplir a través de los planes de mejoramiento y así impactar de forma más efectiva a los grupos de interés identificados. Como señaló Singh (1997) “la administración se encuentra en un estado estable y solo una transformación profunda es necesaria para salir del estado actual y no unos simples remiendos al sistema de gestión actual".

\subsection{Explicación y composición del modelo pro- puesto}

Fase 1: el modelo en su primera fase debe ser definido como un proyecto institucional. El hecho de enmarcar la RS de esta manera les permite ver con más claridad hacia donde se dirigen y que debe hacerse en el camino para llegar a la meta. Por tanto, se vale de algunos elementos que se consideran claves dentro de esta fase:

1. Introducción. En este primer paso se define lo que se pretende con la implementación del modelo desde la misión de cada universidad y su realidad. Aconsejable que se redacte también desde los pilares planteados en el ciclo PHVA (docencia, proyección social, investigación, gestión, medio ambiente, posconflicto) y su trazabilidad.

2. Definición de objetivos. Definir los objetivos que se procuran con el desarrollo del modelo propuesto. De igual manera se recomienda que se determinen desde los pilares del ciclo PHVA y su trazabilidad, pues se supone que debe haber coherencia en el sistema y la interrelación necesaria para obtener los impactos deseados.

3. Organización y administración del proyecto. Es importante darle una estructura. Por tanto, es 
oportuno establecer un comité interdisciplinario donde se definan roles precisos y tareas claras, compuesto (en lo posible) por personas de las diferentes instancias de la universidad. Nótese que no se sugieren personas de los pilares del modelo, sino que para el caso si es necesario que toda la comunidad académica este allí representada, puesto que lo que se busca es la trazabilidad de los planes y proyectos que van a desarrollarse posteriormente.

4. Recursos destinados para el proyecto. Determinar los costos de diseño y puesta en funcionamiento para estimar los recursos suficientes y necesarios para la buena ejecución del modelo, recalcando el hecho que es sistemático; esto significa que va a ser permanente; por tanto se necesita en lo fundamental de: un espacio físico, implementos de oficina y todos los demás insumos que consideren necesarios quienes van a hacer la labor.

Fase 2: definir la situación actual según los siguientes ítems:

5. Levantamiento del diagnóstico. El diagnóstico debe permitir conocer cómo está la universidad en términos de RS, qué se ha hecho en sus diferentes frentes. Se aconseja que se haga con el debido rigor y preferiblemente se cuente con la participación de todas las instancias institucionales, sin dejar de lado a egresados y empleadores; de esta manera se tendrá una plataforma adecuada para poder orientar sus esfuerzos. Para el levantamiento del diagnóstico se pueden emplear los instrumentos acostumbrados: entrevistas, encuestas $u$ otro método que a bien tengan quienes lo van a elaborar y basarse en las tablas de los indicadores, definida en líneas posteriores, como primer paso en el adecuado manejo de la información que se va a acopiar.

6. Concientización y explicación. Concientizar a toda la comunidad sobre la importancia de la RS en el contexto universitario frente a la realidad del país y las tendencias mundiales, a la vez que se familiaricen con la conceptualización y términos nuevos con los que van a tener mucho que ver. Para este propósito se sugiere valerse de: conferencias, seminarios, talleres, entre otros. Este paso se deja a discrecionalidad de quien lidere el proyecto, para que lo integren o no con el paso 9.

7. Definición de los stakeholders internos y externos. La determinación de los grupos de interés, puede hacerse partiendo desde los internos y posteriormente definir los externos; esto depende del criterio del equipo interdisciplinario que esté llevando a cabo la implementación del modelo.

Para los stakeholder externos, un aspecto a tener presente es dejar claro el vínculo que cada uno tiene con la universidad. Acto seguido, puntualizar con cada uno de estos grupos lo que se espera de las partes, todo esto contribuye a fortalecer el diagnóstico, coadyuvando a un adecuado uso del modelo de RSU.

8. Definición de políticas institucionales de RS. Si bien es cierto que cada universidad es autónoma, se sugiere que se tengan en cuenta aspectos como los señalados a continuación para que cada una establezca sus propias políticas: currículos a la luz de la responsabilidad social; interrelación entre las funciones sustantivas; medio ambiente; equidad educativa y de género; derechos humanos; producción del conocimiento; relación con egresados; vínculo con el sector empresarial, gobierno y demás grupos de interés, entre estos uno nuevo, el de los reinsertados; convenios o alianzas estratégicas con otros órganos externos; infraestructura apropiada para personas con algún tipo de discapacidad y demás aspectos que se puedan constituir en política para el buen engranaje del modelo, visto desde una conciencia social que permee todos los rincones de la universidad. También tengan en cuenta al momento de diseñarlas, cuestiones relevantes como: la Declaración Mundial de los Derechos Humanos, los Objetivos del Milenio de la ONU, el posconflicto en Colombia y lo que establece el gobierno nacional a través del 
Ministerio de Educación Nacional y de la Agencia Colombiana para la Reintegración.

Fase 3:

9. Explicación y apropiación del modelo. Es menester de las directivas hacer un plan estratégico para su divulgación. En este paso, el mensaje implícito es que esa conciencia social debe partir desde las cabezas visibles de cada universidad. También, según criterio de quien lidere el proyecto lo puede integrar con el punto 6 .

En este paso se incorpora el ciclo PHVA creado y utilizado para mejorar e implementar procesos y que muy bien se ajusta a los aspectos de la Guía Universia, como criterios para el diseño del modelo propuesto.

Fase 4:

10. Informe final -Informe de autoevaluación y gestión de la RSU- (dirigido a la alta dirección de la universidad). Los resultados de la aplicación del modelo se presentarán en un informe escrito al finalizar cada período establecido, donde se consigne lo planeado, lo ejecutado, lo verificado y las acciones, que vienen siendo los planes de mejoramiento, y los impactos generados en ese período de tiempo, conclusiones y recomendaciones.

El resultado del análisis de cada fase permitirá hacer planes de mejoramiento, teniendo en cuenta: la factibilidad de logros de las propuestas, el análisis de los aspectos institucionales, el logro de las estrategias, así como la identificación de las falencias para su corrección.

11. Informe de Gestión - Rendición de cuentas que la alta dirección debe rendir al público interno y externo (esto también puede aplicar a las universidades privadas).

\subsection{Descripción del ciclo PHVA}

Se empleó el ciclo Shewhart o PHVA, diseñado para ayudar a mejorar los procesos, y se busca sea desarrollado en el marco de las seis dimensiones que se han tenido como parámetros, no de manera independiente, pues si bien hay proyectos y programas que se llevan a cabo en cada división, lo que se plantea es la integralidad o transversalidad de los elementos que la componen.

Planear: se seleccionan planes y programas para luego determinar las actividades a seguir para su desarrollo, previa definición de los objetivos. Importante aclarar que son los objetivos de cada plan y que por razón a la lógica, deben ser coherentes con los de la institución y los de la implementación del modelo establecidos en la fase 1.

En este punto se recomienda dar una mirada a la situación actual del tema que se vaya a trabajar (se recuerda que estos proceden de la etapa de VERIFICACIÓN), en el que se obtengan datos y se haga su revisión, para luego analizar las causas y determinar las acciones correctivas. Al efecto se recomienda hacer un análisis causa-efecto, establecer hipótesis si es necesario, verificar las causas más probables y determinar acciones correctivas (a corto plazo o remediadoras y a largo plazo o preventivas).

Hacer: se ponen en práctica las acciones correctivas. Para mejores resultados se recomienda capacitar a quienes van a encargarse de estas acciones y preferiblemente seleccionar a personas con experiencia sobre lo que se va a emprender. De igual manera hay que considerar si se requiere de un equipo interdisciplinario para este fin.

Verificar: Esta etapa se constituye quizás en la más importante al momento de implementar el modelo por primera vez. La verificación se hace de acuerdo a los indicadores. De esta etapa se desprenden los aspectos susceptibles a convertirse en planes de mejoramiento, los cuales son los que entran a la etapa de planeación, y es en ese mo- 
mento donde comienza a dinamizarse el esquema circular del ciclo. Se espera que, a medida que gira el ciclo, las tablas de indicadores tengan más respuestas positivas y sus índices numéricos crezcan.

Actuar: emprender las acciones apropiadas para institucionalizar el cambio. Se concluye y establecen estrategias para que los proyectos y programas que dieron óptimos resultados, se mantengan e inclusive estén sujetos a mejoras y cambios. Evidentemente habrán planes que deben termi- nar en un momento determinado para darle paso a otros de acuerdo a las tendencias y las realidades que se vayan presentando en el ámbito de influencia, lo cual muestra una vez más el dinamismo del modelo.

En la figura 1 se puede observar la dinámica del ciclo, la transversalidad e interdisciplinariedad de los planes y proyectos en: docencia, proyección social, investigación, gestión, medio ambiente y posconflicto.

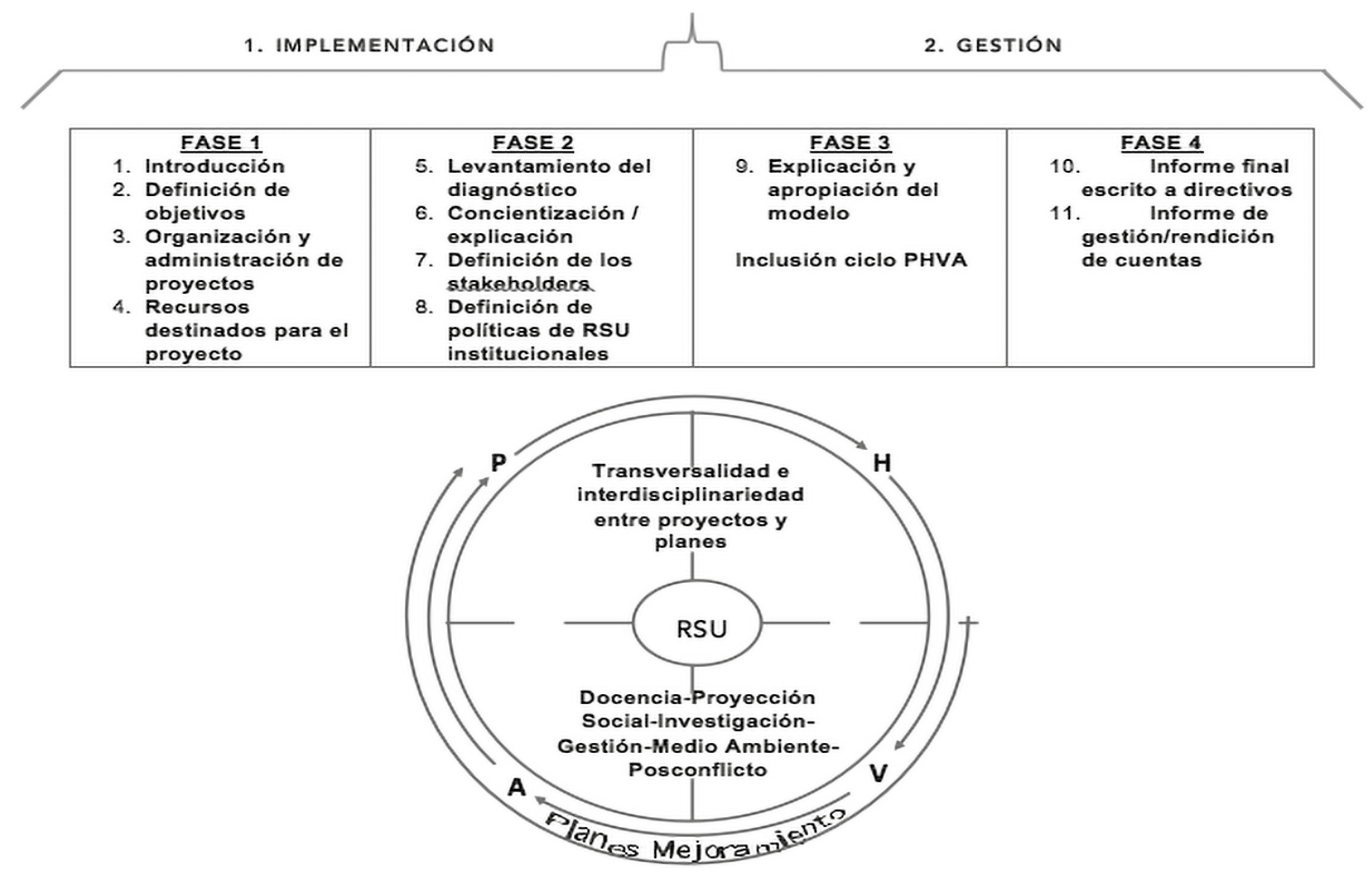

Figura 1. Modelo implementación RSU.

\subsection{Indicadores de RSU}

La universidad al igual que otras organizaciones genera impactos, por lo que debe evaluarse y auto-regularse en forma permanente. En este sentido, es importante considerar que "los procesos de evaluación requieren de propuestas participativas de las comunidades, a partir de las cuales puedan construirse redes de solidaridad y apoyo, basadas en el principio de la Responsabilidad y la Libertad" (Cardona \& Zambrano, sf).

Vista la utilidad de los indicadores, de acuerdo con Vallaeys (2008) surgen las preguntas ¿cómo medir la RSU? ¿cómo aplicar los indicadores? o ¿cómo determinarlos?. Estos cuestionamientos son el punto de partida para empezar un trabajo juicioso y es que al momento de proceder a ejecutar la 
tarea se presentan inconvenientes, porque existe muy poco sobre sistemas de gestión o de aplicación y evaluación de modelos de RS. Previo a esto, se determinan el propósito y los objetivos del porqué se hace la autoevaluación, cuál va a ser el método empleado, y según la National Service-Learning Clearinghouse (2009, citado por Baca, 2015), si va a ser por estamentos o en toda la universidad. Igualmente es relevante saber si va a ser cuantitativa o cualitativa, para otros de entrada o salida, o ambos tipos; el uso de los dos puede ser útil, esto se da de acuerdo con los objetivos perseguidos en la medición.

En el caso de este modelo, se establece el empleo de indicadores cualitativos resultado de la aplicación de los indicadores cuantitativos. Adicionalmente, para garantizar la efectividad del sistema de autoevaluación y gestión de RSU, se sugiere que para un mayor control de los procesos la aplicación sea anual.

\section{Criterios para determinar los indicadores}

Para la selección de los indicadores se recomienda tener en cuenta los criterios definidos por AUSJAL (2014), que sirven para definirlos independiente del método que se vaya a utilizar en la medición de la RSU, a saber: pertinencia y claridad de los indicadores seleccionados; agrupamiento de los indicadores en torno a cinco impactos; combinación de parámetros cuantitativos (numéricos y porcentuales), y cualitativos (de percepción y valoración subjetiva); viabilidad en la recolección de la información, adecuándola a la disponibilidad y los procedimientos habituales de generación y reporte de información de las universidades; razonabilidad y coherencia entre los costos derivados del sistema de medición por indicadores propuesto $y$ el volumen total de recursos destinados por las universidades a acciones vinculadas a la RSU; cantidad reducida de indicadores, a fin de facilitar el proceso de relevamiento de datos, sistematización y comunicación de los mismos; comparabilidad de los datos a fin de favorecer la medición entre las diferentes universidades.

\section{Descripción y uso de las tablas de indicadores}

"Los indicadores de RSU que se presentan se convierten en una alternativa para transparentar la gestión socialmente responsable de las instituciones universitarias de forma específica..., teniendo como sustento base que si bien el modelo socialmente responsable no responde, ni debe responder a una normativa legal impuesta, su gestión debe ser transparentada y acreditada" (Valarezo et al., 2013).

En el modelo planteado, se mantiene la pertinencia y se parte de los seis elementos mencionados previamente, los que a su vez, se organizan por variables como se observa en la tabla 1.

Tabla 1. Indicadores de Evaluación propuestos.

\begin{tabular}{ll}
\hline Variable & Indicadores \\
\hline $\begin{array}{l}\text { Docencia } \\
\text { Prácticas académi- } \\
\text { cas }\end{array}$ & $\begin{array}{l}\text { D1. Las prácticas académicas están dirigidas a aportar soluciones a problemas sociales de los } \\
\text { grupos de interés definidos por la universidad. }\end{array}$ \\
$\begin{array}{l}\text { Contenido curri- } \\
\text { cular }\end{array}$ & D2. Componentes temáticos con contenidos explícitos de Responsabilidad Social. \\
$\begin{array}{l}\text { Convenios } \\
\text { Interdisciplinarie- } \\
\text { dad }\end{array}$ & $\begin{array}{l}\text { D3. Tiene convenios con comunidades para realizar docencia e investigación en ellas. } \\
\text { estudiantes de distintas disciplinas formen parte de actividades, proyectos o programas con } \\
\text { enfoque de RS. }\end{array}$
\end{tabular}

Proyección Social 


\begin{tabular}{|c|c|}
\hline Variable & Indicadores \\
\hline & $\begin{array}{l}\text { PS1. Se reúne con actores claves de desarrollo social (Estado, ONGs, empresas, organismos in- } \\
\text { ternacionales), para tratar temas sociales diagnosticados y trabajar mancomunadamente para } \\
\text { resolverlos. }\end{array}$ \\
\hline & $\begin{array}{l}\text { PS2. Tiene políticas que beneficien a grupos poco representados o marginados } \nabla \text { (población } \\
\text { indígena, minoría racial, personas con discapacidad física o mental, padres } \text { đde familia mayo- } \\
\text { res de } 45 \text { años, personas con } \mathrm{VIH} \text {, ex convictos, etc.), y pueden estos acceder a los programas } \\
\text { académicos. }\end{array}$ \\
\hline
\end{tabular}

Investigación

11. Existen líneas de investigación que respondan a problemas sociales de las comunidades vulnerables, contribuyendo a una mayor equidad social.

12. Existen políticas que determinen que los estudiantes de pregrado deben practicar la investigación en distintas asignaturas a lo largo de su carrera.

Gestión

Políticas/normas/ G1. En el ADN de las políticas y estrategias institucionales esta la RS.

reglas G2.凶Capacita a los docentes y al personal administrativo en RS.

Ética

G3. Existe un código de ética y es conocido por la comunidad académica y resocializado periódicamente. Además cuentan con asignaturas de ética en cada carrera que se refieran al código.

G4. La declaración de la misión, visión y estrategia contemplan aspectos éticos.

Proveedores

G5. Además de criterios básicos de respeto a la legislación, sus normas de selección y evaluación de proveedores incluyen criterios específicos de RS, como los contemplados en prohibición del trabajo infantil (Convenio 138 de la OIT), prácticas ambientalistas, apoyo a iniciativas sociales, relaciones de trabajo adecuadas.

G6. Exige que sus proveedores reproduzcan sus exigencias en lo que se refiere a la responsabilidad social para sus respectivos proveedores y que monitoricen esos criterios periódicamente.

Estudiantes

TIC

G7. Tiene políticas para la contratación de estudiantes.

G8. La universidad invierte en nuevas tecnologías que benefician a docentes, estudiantes, administrativos.

Medio Ambiente

A1. Existen programas a través de los cuales: se concientiza, educa y adelantan acciones concretas para el cuidado del MA.

A2. Tiene líneas de investigación orientadas a estudiar el impacto ambiental que genera la universidad, como resultado de las actividades e investigaciones ambientales y soluciones técnicas que dan respuesta a problemas ecológicos.

A3. Se suma a la Década de las Naciones Unidas a través de la agenda 2030 para el desarrollo sostenible y ejerce un liderazgo social a favor de esa causa.

A4. Trata de practicar entre todos sus miembros los "4 RE": REutilizar, REciclar, REducir, REspetar.

Posconflicto

Docencia

PC1. Tiene incluido en los currículos de pregrado y postgrado temas relacionados con la paz y posconflicto en Colombia. 


\begin{tabular}{ll}
\hline Variable & Indicadores \\
\hline Proyección social & PC2. Existen programas de extensión orientados a los reinsertados. \\
& $\begin{array}{l}\text { PC3. Se reúne con actores claves de desarrollo social (Estado, ONGs, empresas, organismos in- } \\
\text { ternacionales), para tratar el tema de los reinsertados y trabajar mancomunadamente a través } \\
\text { de convenios. }\end{array}$ \\
& $\begin{array}{l}\text { PC4. Adelanta investigaciones atinentes al tema de la paz en Colombia: antes, durante y des- } \\
\text { pués?. }\end{array}$ \\
& PC5. Cuenta con una línea de investigación que aborde el posconflicto. \\
& PC6. Proyectos o programas que adelanta o construyó la universidad para vincularse en la eta- \\
& pa de posconflicto con las FARC. \\
& PC7. Tiene propuestas para el posconflicto. \\
& PC8. Han hecho algún tipo de preparación sicológica y social para que la comunidad académi- \\
& Ca esté preparada para la integración con los reinsertados. \\
& PC9. Es posible que se incorporen personas reinsertadas a la nómina.
\end{tabular}

La tabla 2 es donde se migrará el contenido de la tabla 1, según la actividad sustantiva; esto significa que se obtendrán seis tablas de indicadores que se trabajarán de forma independiente, con el propósito de efectuar una evaluación adecuada y que sus resultados sean los suficientemente consistentes para los planes de mejora.

Los puntos a considerar para el adecuado uso de la tabla 2 son: se compone de tres columnas básicas denominadas: variable, indicadores, criterios de evaluación, este último se subdivide en cualitativos y cuantitativos, los que a su vez se subdividen en -SI -NO - Cantidades y -Porcentajes respectivamente y uno último de No Aplica, el cual da paso a cualquier particularidad que debe ser sustentada al final de cada tabla.

La primera columna, "VARIABLES", como su nombre lo indica la componen las variables derivadas de la columna de INDICADORES. En cuanto a las cantidades y porcentajes de la casilla de criterios de evaluación cuantitativa, deben estar respaldados previamente por un SI de la casilla de evaluación cualitativa. La respuesta en cantidades o porcentajes depende del criterio del equipo evaluador, quienes a su vez deben estar soportados de las pruebas documentales que den fe de los afirmados. Las respuestas con No evidentemente no van a tener respaldo numérico, por lo que al final de cada tabla debe ser igualmente explicado cada ítem y de acuerdo al tema que se trate entrará a formar parte del ciclo PHVA como proyecto. Luego que se diligencie cada tabla se hará la sumatoria de los $\mathrm{SI}$, de los NO, de las cantidades, de los porcentajes y de los NA, para hacer un balance de la situación real de la universidad, que de paso sirve de diagnóstico al ser diligenciadas las tablas por primera vez. 
Tabla 2. Migración y procesamiento de la información.

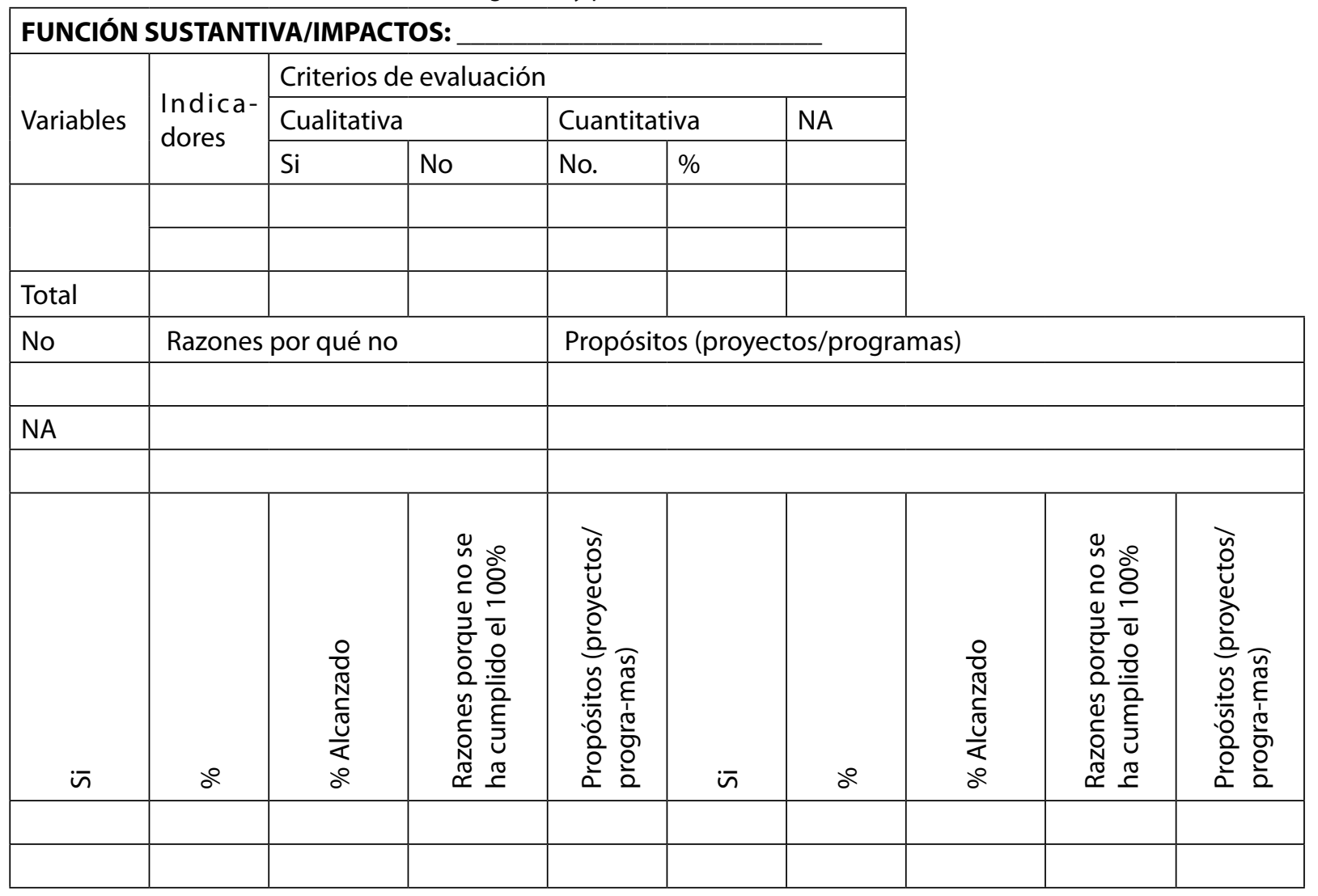

\section{Conclusiones}

La falta de implementación de la responsabilidad social en un amplio número de universidades colombianas, se debe a que no se ha tomado real consciencia de su importancia y consecuencias positivas, pero además, porque no se cuenta con una metodología de cómo hacerlo.

Para ello es preciso contar con un modelo para su implementación, esto en aras de ofrecerles herramientas para que su labor sea más sencilla y se motiven a emprender el camino. Así, se hace necesario contar con un banco de indicadores bien estructurado que forme parte de la metodología o modelo para lograr los resultados esperados.

La RSU debe formar parte de la estructura administrativa de las universidades. Esto significa que debe contar con un equipo profesional encarga- do de su implementación y puesta en marcha de cada proyecto, programa o actividades que de esta se deriven, garantizando que los stakeholders involucrados sean portadores de los buenos resultados de su gestión.

Las universidades deben poner especial atención a los stakeholder que van surgiendo, producto de los cambios sociales, económicos, políticos, del estado, y encaminar esfuerzos a satisfacer sus expectativas, reconociendo que de esta manera se auto benefician y a la sociedad en su conjunto.

\section{Referencias}

Aristimuño, M. (2012). La valoración de la responsabilidad social universitaria: dimensiones e indicadores para su abordaje. Revista Copérnico Arbitrada de Divulgación Científica, 8 (16), 23-29. 
Recuperado de: http://copernico.uneg.edu.ve/numeros/c16/c16_art03.pdf

Asociación Colombiana de Universidades (2010). Políticas para la educación superior en Colombia 2010 - 2014: Hacia una nueva dinámica social de la educación superior. Revista El Pensamiento Universitario, (19), 34-38. Recuperado de: https://www.cna.gov. co/1741/articles-311056_ResponsabilidadSocial.pdf

Asociación de Universidades Confiadas a la Compañía de Jesús en América Latina (2014). Proyecto de fortalecimiento institucional de la responsabilidad social de las universidades (RSU) para AUSJAL. Red Homólogos de RSU - AUSJAL 2014. Recuperado de: https:// issuu.com/ausjal/docs/carta_ausjal_42

Baca, H. (2015). La responsabilidad social universitaria: propuesta conceptual y medición en el ámbito de una universidad privada de Lima - Perú (Tesis doctoral). Sevilla, España: Universidad de Sevilla.

Cardona, D., \& Zambrano, R. (s/f). En los límites entre el impacto de las propuestas de intervención y la responsabilidad social universitaria. Recuperado de: http:// bit.ly/2DqANXT

Hernández, R., Fernández, C., \& Baptista, P. (2010). Metodología de la Investigación (5a Ed.). México: McGraw Hill Educación.

Lerma, H. D. (2016). Metodología de la investigación. Pereira, Colombia: Ecoe Ediciones.

Lizarazo, T. (19 de mayo de 2015). ¿Qué papel tienen las universidades dentro del proceso de paz?. Configurar escenarios para el posconflicto es uno de los objetivos de la academia en pro de la paz. El Tiempo. Recuperado de: $\mathrm{http}: / /$ bit.ly/2rxhsTA

Pagano, R. R. (2000). Estadística para las ciencias del comportamiento. Madrid, España: International Thompson.

Pérez, F. (2016). El papel de la educación en el posconflicto. Corporación Viva la Ciudadanía. Recuperado de: http://bit.ly/2DsVimP
Sarmiento, J. M. (2009). El ciclo PHVA:Una herramienta para la mejora continua. Recuperado de: http://nolimitsquality.blogspot.com/2009/11/el-ciclo-phva. html

Singh-Soin, S. (1997). Control de calidad total. México: McGraw Hill.

Universia (2017). Tipos de investigación: Descriptiva, exploratoria y explicativa. Recuperado de: $\quad$ http://noticias.universia.cr/educacion/noticia/2017/09/04/1155475/tipos-investigacion-descriptiva-exploratoria-explicativa.pdf

Valarezo-González, K., Sarango-Lalangui, P., Salcedo-Viteri, K., Valdiviezo-Abad, C., Sánchez-Farfán, L., \& Marín-Gutiérrez, I., (2013). Gestión universitaria ética y responsable. Indicadores de RSU. En Actas - V Congreso Internacional Latina de Comunicación Social - VCILCS. Recuperado de: http://www.revistalatinacs. org/13SLCS/2013_actas.html

Vallaeys, F. (2006). Breve marco teórico de responsabilidad social universitaria. Recuperado de: https://www. econo.unlp.edu.ar/frontend/media/86/10986/10ec2d7e617a62eba0696821196efeae.pdf

Vallaeys, F. (2008)."Responsabilidad social universitaria": una nueva filosofía de gestión ética e inteligente para las universidades. Revista Educación Superior y Sociedad, 13 (2), 191-220. Recuperado de: http:// ess.iesalc.unesco.org.ve/ess3/index.php/ess/article/ view/47

Vallaeys, F., \& Carrizo, L. (2011). Hacia la construcción de indicadores de responsabilidad social universitaria. Recuperado de: http://bit.ly/2ry7ls8

Yfarraguerri, L. (2014). Necesidad de indicadores de medición de la responsabilidad social en las universidades. Revista Daena: International Journal of Good Conscience, 9 (1), 142-157. Recuperado de: http:// spentamexico.org/v9-n1/A13.9(1)142-157.pdf 